\title{
Diffusiophoresis of concentrated suspensions of spherical particles with identical ionic diffusion velocities
}

\author{
James Lou, Yan-Ying He, Eric Lee* \\ Department of Chemical Engineering, National Taiwan University, Taiwan 10617, Taipei \\ Received 15 November 2005; accepted 20 January 2006 \\ Available online 9 March 2006
}

\begin{abstract}
Diffusiophoresis of concentrated suspensions of spherical particles subject to a small electrolyte gradient is analyzed theoretically at arbitrary levels of zeta potential and double-layer thickness. The Kuwabara unit cell model is adopted to describe the system under consideration. The effect of double-layer polarization is taken into account. It is found that the diffusiophoretic mobility exhibits a local maximum as well as a local minimum with varying zeta potential or double-layer thickness, similar to the corresponding dilute dispersion. The direction of the particle movement may even change back and forth. The previous low-zeta-potential approach is found to significantly overestimate the diffusiophoretic mobility as the zeta potential goes high. The deviation may be several fold sometimes. The effect of the volume fraction ratio of colloids is also examined. The higher the ratio, the lower the mobility.
\end{abstract}

(c) 2006 Elsevier Inc. All rights reserved.

Keywords: Diffusiophoresis; Polarization effect; Kuwabara unit cell model; Arbitrary zeta potential

\section{Introduction}

The electrokinetic behavior of colloidal particles dispersed in a continuous medium is of great practical interest. When an external electric field is applied to a uniform electrolyte solution containing charged colloidal particles, the resulting migration of colloidal particles is called "electrophoresis" [1,2]. This well-known phenomenon has been treated extensively in the past literature [3-9]. However, the corresponding "diffusiophoresis" has received very limited attention so far. If the concentration of electrolyte solute is somehow originally nonuniform in the solution, an induced electric force will arise, due to this macroscopic concentration gradient of electrolyte solute. Hence the colloidal particles will migrate nonetheless, even though the applied electric field is absent.

Deryagin et al. $[10,11]$ first noticed this electrokinetic phenomenon and proposed the diffusiophoresis mechanism to explain it. They found that diffusiophoresis was the underlying mechanism for industrial processes such as ionic deposition

\footnotetext{
* Corresponding author.

E-mail address: ericlee@ntu.edu.tw (E. Lee).
}

[10-12]. When a solid shape containing a salt was immersed in a latex solution, the growth rate of the latex film on the solid shape would be comparable to that from the electrodeposition process, where hundreds of volts are required. In other words, the new process was able to achieve about the same growth rate of latex films on a substrate without the application of any electric field $[11,12]$. A corresponding theoretical analysis in electrolyte solution was carried out by Dukhin and Deryagin $[10,13]$ and experimentally verified by Korotkova and co-workers [10]. They proposed that the diffusiophoretic velocity of a charged particle subject to a constant concentration gradient $\nabla n^{\infty}$ in an unbounded solution of a symmetrically binary electrolyte is

$\mathbf{U}^{*}=\frac{\varepsilon \zeta}{\mu} \frac{k_{\mathrm{B}} T}{z e} \frac{\nabla n^{\infty}}{n_{0}}\left[\beta+\bar{\zeta}^{-1} \ln (\cosh \bar{\zeta})\right]$,

where $\bar{\zeta}=z e \zeta / 4 k_{\mathrm{B}} T$, ze is the charge carried by a single ion, $k_{\mathrm{B}}$ is the Boltzmann constant, $T$ is the temperature, $\zeta$ is the zeta potential of the particle surface, $\varepsilon$ is the fluid permittivity, $\mu$ is the fluid viscosity, and $n_{0}$ is the macroscopic electrolyte concentration measured without the particle and concentration gradient. Note that $\beta=\left(D_{1}-D_{2}\right) /\left(D_{1}+D_{2}\right)$, where $D_{1}$ and $D_{2}$ are the diffusion coefficients for cations and anions, respec- 
tively, is a dimensionless index indicating the relative diffusion velocities of cations and anions. $\beta$ is an experimentally measurable property of the specific electrolyte solution under consideration. For example, $\beta=0$ for $\mathrm{KCl}, \beta=-0.2$ for $\mathrm{NaCl}$, and $\beta=0.64$ for $\mathrm{HCl}$. Note also that $\beta=0$ implies identical diffusion velocities of anions and cations. Based on the GouyChapman model [1,2], Anderson [14] was able to predict the diffusiophoretic mobility of a charged spherical particle when the double-layer thickness and the zeta potential were small. He found that the diffusiophoretic mobility in an electrolyte solution was determined essentially by three factors: the zeta potential of the particle surface, the relative diffusion velocities of ions $(\beta)$, and fluid properties such as viscosity, temperature, etc. Prieve and co-workers [15-18] further loosened the restriction of low zeta potential in their theoretical analyses and observed strong evidence supporting their proposed diffusiophoretic mechanism with experimental data. Concentration gradients of electrolytes were established and measured in their experimental setup, making use of a porous membrane [18]. Lechnick and Joseph [19,20] further extended Deryagin's results to ternary-component systems and constructed ternary diffusion coefficients both theoretically and experimentally. With the aid of the Maxwell-Stefan equation, they concluded that the Stokes-Einstein theory was not suitable to predict the binary diffusion coefficient for a charged particle in electrolyte solution. Denisov and Zharkikh [21] solved the corresponding problem with arbitrary double-layer thickness. Afterwards Prieve and Roman [22] calculated diffusiophoretic mobility with arbitrary double-layer thickness, using the same numerical method as employed by O'Brien and White [6]. In their study, they compared the difference between cases where $\beta=0$ $(\mathrm{KCl})$ and $\beta \neq 0(\mathrm{NaCl})$. They also concluded that the diffusiophoretic motion of colloidal particles cannot be totally predicted by thermodynamic forces in electrolyte solution [23]. Dukhin [24] provided a thorough physical analysis later on. He figured that the external concentration had also induced a concentration and electrical dipole moment. This nonequilibrium ionic distribution might play an important role in diffusiophoresis. Baygents and Saville [25] studied numerically the diffusiophoresis of a droplet or a small bubble suspended in an electrolyte solution with the finite element method. Misra et al. [26] explored the effect of the soft layer on charged colloids in diffusiophoresis. Following a similar treatment, Kosmulski and Matijevic [27] proposed another mechanism named "solvophoresis." They found that different solvents might have similar results even though the solutes were the same.

From this literature review, we noticed that essentially all the studies on diffusiophoresis, analytical or numerical, were confined to very dilute dispersion systems. When the concentration of colloidal particles is high, however, the existence of neighboring particles can no longer be ignored. This boundary effect will be felt by all the colloids in the solution. Hence the appropriate determination of associated boundary conditions on the virtual surface, representing the interface between neighboring particles, is essential for a successful theoretical approach. As a result, theoretical studies on diffusiophoresis have been focusing on systems with boundaries, virtual or physical, in re- cent years. The existence of a physical boundary is in itself an interesting classic problem. For example, Keh and co-workers $[28,29]$ considered the diffusiophoresis for a colloidal sphere in a nonelectrolyte or electrolyte solutions near a planar boundary. They showed that the retardation effect produced by a planar boundary might decelerate the motion of the particle. In addition, Wei and Keh [30] and Keh and Wei [31] predicted the diffusiophoretic behavior in a suspension using both the Happel model [32] and the Kuwabara [33] unit-cell model. They showed that the Kuwabara unit-cell model was more appropriate than the Happel model for the system under consideration. In recent years, Keh and Ma [34,35] also obtained diffusioosmotic flow near a plane boundary. However, their results were restricted to low surface potential, with no account of the convection of ions.

What we present here is a study of the diffusiophoretic behavior of spherical particles dispersed in an electrolyte solution, with arbitrary surface potentials and arbitrary double-layer thickness. In fact, diffusiophoresis exists not only in electrolyte solutions [36], but also in nonelectrolyte solutions [37]. However, the electrokinetic phenomenon of diffusiophoresis in electrolyte solution is much more interesting and complicated. In our analysis, we focus on the situation where the mobility of the two ions is identical in the electrolyte solution $(\beta=0)$. Compared with the results in the earlier literature, our study of the diffusiophoresis of concentrated colloidal dispersion takes into account the arbitrary effects of zeta potential, double-layer thickness, and volume fractions of the particle. To solve the resulting general electrokinetic equations, which are highly nonlinear, and the powerful pseudo-spectral method [8,38-40] is employed in this study.

\section{Theory}

We consider the diffusiophoretic behavior of concentrated spherical particles of radius $a$ in a solution that contains $z_{1}: z_{2}$ electrolytes, $z_{1}$ and $z_{2}$ being respectively the valences of cations and anions. The Kuwabara [33] unit-cell model is adopted as the basis for subsequent analysis. Referring to Fig. 1, a uniform concentration gradient $\nabla \mathbf{n}_{0}$ is applied to the system in the $z$ direction. The radius of each particle is $a$. Each individual particle is considered as surrounded by a concentric spherical shell of electrolyte solution phase of radius $b$, and moves upward with a constant velocity $\mathbf{U}$ due to the concentration gradient. As a result, it is most convenient to use spherical coordinates $(r, \theta, \phi)$ in our system. The electroneutrality constraint in the bulk liquid phase requires that $n_{20}=n_{10} / \alpha, n_{10}$ and $n_{20}$ be the bulk concentrations of cations and anions, and $\alpha=-z_{2} / z_{1}$.

It is assumed that the physical properties of the liquid phase take their macroscopic values. Also, the shape of the colloidal particle remains spherical when it is migrating in the fluid, which is generally true for low-Reynolds-number situations. It is further assumed that the concentration of solute is only slightly nonuniform over the length scale $a$; that is, $a\left|\nabla \mathbf{n}_{0}\right| \ll$ $n_{0}$. Under these conditions, the diffusiophoretic motion is governed by the well-known electrokinetic equations, which we shall elaborate as follows. 


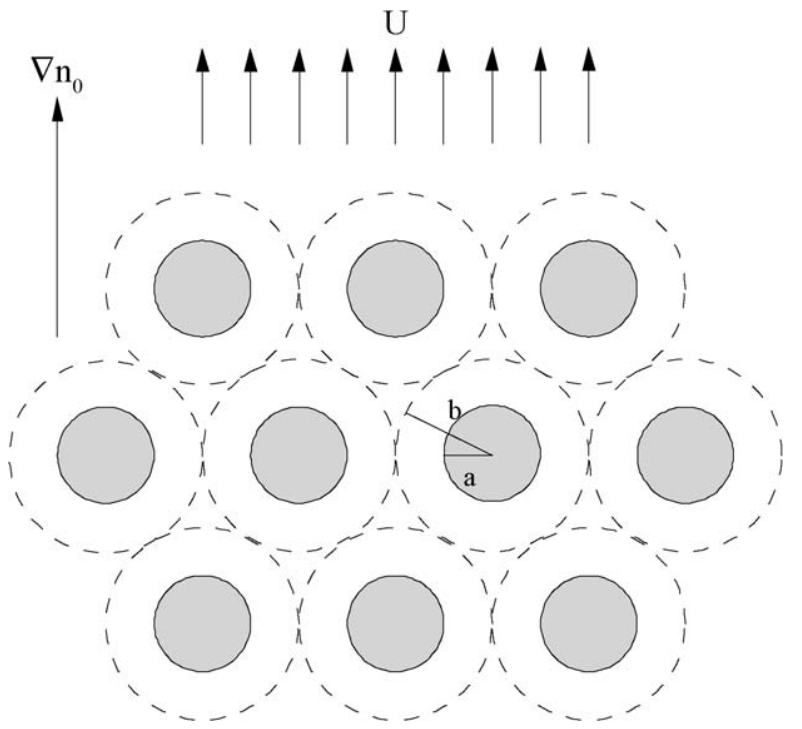

Fig. 1. Schematic representation of the system under consideration.

First we assume that the electrical potential $\phi$ of our system under consideration can be described by the Poisson equation,

$\nabla^{2} \phi=-\frac{\rho}{\varepsilon}=-\sum_{j=1}^{2} \frac{z_{j} e n_{j}}{\varepsilon}$,

where $\rho$ and $\varepsilon$ are respectively the space charge density and the permittivity of the solution, $e$ is the elementary charge, and $n_{j}$ and $z_{j}$ are respectively the number concentration and the valence of ionic species $j$. Moreover, the flow field is governed by the Navier-Stokes equation in the creeping flow region, together with an incompressibility constraint,

$\nabla \cdot \mathbf{v}=0$

$\mu \nabla^{2} \mathbf{v}-\nabla p-\rho \nabla \phi=0$,

where $p$ and $\mu$ are respectively the pressure and the viscosity of the fluid. The ionic concentration $n_{j}$ is governed by the conservation of ion species,

$\frac{k T}{D_{j}}\left[\nabla^{2} n_{j}+\frac{z_{j} e}{k T}\left(\nabla n_{j} \cdot \nabla \phi+n_{j} \nabla^{2} \phi\right)\right]-\nabla n_{j} \cdot \mathbf{v}=0$,

where $D_{j}$ is the diffusion coefficient of ion species $j, \lambda_{j}$ is the diffusion coefficient for ion $j$, and $z_{j} e$ is the charge carried by ion $j$.

We nondimensionalize the above quantities further to facilitate subsequent treatments. Let $\phi_{\mathrm{e}}^{*}=\phi_{\mathrm{e}} / \zeta$, where $\phi_{\mathrm{e}}$ means the equilibrium electric potential in the solution, with the absence of the applied electric field, $r^{*}=r / a$, and $n_{j}^{*}=n_{j} / n_{10}$, where $\zeta$ represents the zeta potential on the shear plane. The conventional standard electrokinetic model is adopted in this study, in that no surface conductivity is considered here. The surface conductivity, however, is a potentially important factor for the thorough understanding of the electrokinetic phenomena, as pointed out by Dukhin, Delgado et al. [41,42]. Superscripts on all the quantities indicate corresponding dimensionless forms. If the surface potential is uniform on the colloidal surface, with the introduction of the convection term in Eq. (5), the ion distribution in the electrolyte solution can be described in a form similar to the Boltzmann distribution,

$n_{j}=n_{j 0} \exp \left(-\frac{z_{j} e}{k_{\mathrm{B}} T}\left(\phi_{\mathrm{e}}+\delta \phi+g_{j}\right)\right)$,

where $n_{j 0}$ is the bulk concentration of the species $j, \delta \phi$ the induced electric potential due to the difference of ion mobilities, and $g_{j}$ a perturbed potential adopted to describe the doublelayer polarization. Note that to analyze separately the effects of double-layer polarization and induced electric field, we have decomposed $\phi$ into $\phi_{\mathrm{e}}, \delta \phi$, and $g_{j}$; that is, $\phi=\phi_{\mathrm{e}}+\delta \phi+g_{j}$. Note also that Eq. (6) is just a convenient analog, the generality of polarization effect due to the fact that inclusion of convection term in the ionic flux equation, $g_{j}$, is intact [40]. Therefore, the equilibrium potential can be determined as

$\nabla^{2} \phi_{\mathrm{e}}^{*}=-\frac{(\kappa a)^{2}}{(1+\alpha) \phi_{\mathrm{r}}}\left[\exp \left(-\phi_{\mathrm{r}} \phi_{\mathrm{e}}^{*}\right)-\exp \left(\alpha \phi_{\mathrm{r}} \phi_{\mathrm{e}}^{*}\right)\right]$,

where the inverse Debye length $\kappa$ and the scaled zeta potential $\phi_{\mathrm{r}}$ are defined respectively by

$\kappa=\left[\sum_{j=1}^{2} n_{j 0}\left(e z_{j}\right)^{2} / \varepsilon k_{\mathrm{B}} T\right]^{1 / 2}$,

$\phi_{\mathrm{r}}=\frac{\zeta}{z_{1} e / k T}$.

The boundary conditions for $\phi_{\mathrm{e}}^{*}$ are:

$\phi_{\mathrm{e}}^{*}=1 \quad$ at $r^{*}=1$,

$\frac{\partial \phi_{\mathrm{e}}^{*}}{\partial r^{*}}=0 \quad$ at $r^{*}=\frac{b}{a}$.

Equation (10) means that the surface potential of the particle at equilibrium remains constant and Eq. (11) implies that the unit cell as a whole is electrically neutral; thus there is no electric current between adjacent cells.

Substituting Eq. (6) into Eq. (2), we obtain the governing equation of induced electric potential from Eqs. (2) and (7) in the dimensionless form

$$
\begin{aligned}
\nabla^{* 2} \delta \phi^{*}= & -\frac{(\kappa a)^{2}}{(1+\alpha) \phi_{\mathrm{r}}}\left\{\exp \left[-\phi_{\mathrm{r}}\left(\phi_{\mathrm{e}}^{*}+\delta \phi^{*}+g_{1}^{*}\right)\right]\right. \\
& \left.-\exp \left[\alpha \phi_{\mathrm{r}}\left(\phi_{\mathrm{e}}^{*}+\delta \phi^{*}+g_{2}^{*}\right)\right]\right\} \\
& +\frac{(\kappa a)^{2}}{(1+\alpha) \phi_{\mathrm{r}}}\left[\exp \left(-\phi_{\mathrm{r}} \phi_{\mathrm{e}}^{*}\right)-\exp \left(\alpha \phi_{\mathrm{r}} \phi_{\mathrm{e}}^{*}\right)\right],
\end{aligned}
$$

and the corresponding boundary conditions are:

$\frac{\partial \delta \phi^{*}}{\partial r^{*}}=0 \quad$ at $r^{*}=1$,

$\delta \phi^{*}=0 \quad$ at $r^{*}=\frac{b}{a}$,

where $\delta \phi^{*}=\delta \phi / \zeta$. The first condition represents that it is dielectric inside the colloidal particle and the other one implies that the net flux for cations and anions is zero across the outer virtual cell. These are also the boundary conditions adopted by Keh and Wei [31]. To simplify the system and focus on issues 
of major interest, we consider the situation where $\beta=0$ for the time being in this paper.

The conservation equation of ions, Eq. (5), is converted into dimensionless form by substituting Eqs. (2) and (6) into it,

$$
\begin{aligned}
& \nabla^{* 2} g_{j}^{*}-\phi_{\mathrm{r}} \nabla^{*} \phi_{\mathrm{e}}^{*} \cdot \nabla^{*} g_{j}^{*} \\
& \quad-\phi_{\mathrm{r}}^{2} P e_{j} \mathbf{v}^{*} \cdot\left(\nabla^{*} \phi_{\mathrm{e}}^{*}+\nabla^{*} \delta \phi^{*}+\nabla^{*} g_{j}^{*}\right) \\
& \quad-\phi_{\mathrm{r}}\left(\nabla^{*} \delta \phi^{*}+\nabla^{*} g_{j}^{*}\right) \cdot \nabla^{*} g_{j}^{*}=0,
\end{aligned}
$$

where $g_{j}^{*}=g_{j} / \zeta$ and $P e_{j}$ is the corresponding Peclet number of ion $j$, taking into account convection. We assume that the colloidal particle is impermeable to ions, and there is an ionic concentration gradient across the outer virtual surface of the unit cell. Therefore,

$$
\begin{aligned}
& \frac{\partial g_{1}^{*}}{\partial r^{*}}=\frac{\partial g_{2}^{*}}{\partial r^{*}}=0 \quad \text { at } r^{*}=1, \\
& \left\{\begin{array}{l}
\left(\delta \phi^{*}+g_{1}^{*}\right)=-\frac{1}{\phi_{\mathrm{r}}}\left(\nabla^{*} n_{0}^{*}\right) \\
\left(\delta \phi^{*}+g_{2}^{*}\right)=\frac{1}{\alpha \phi_{\mathrm{r}}}\left(\nabla^{*} n_{0}^{*}\right)
\end{array}\right\} \quad \text { at } r^{*}=\frac{b}{a} .
\end{aligned}
$$

Taking curl on Eq. (4) and introducing the stream function in the spherical coordinates, we get rid of the continuity equation and obtain

$$
\begin{aligned}
E^{* 4} \psi^{*}= & -\frac{(\kappa a)^{2}}{1+\alpha}\left\{\left[n_{1}^{*} \frac{\partial g_{1}^{*}}{\partial r^{*}}+n_{2}^{*} \frac{\partial g_{2}^{*}}{\partial r^{*}}\left(\alpha n_{2}^{*}\right)\right] \frac{\partial \phi^{*}}{\partial \theta}\right. \\
& \left.-\left[n_{1}^{*} \frac{\partial g_{1}^{*}}{\partial \theta^{*}}+n_{2}^{*} \frac{\partial g_{2}^{*}}{\partial \theta^{*}}\left(\alpha n_{2}^{*}\right)\right] \frac{\partial \phi^{*}}{\partial r^{*}}\right\} \sin \theta,
\end{aligned}
$$

where $\psi^{*}$ is the scaled stream function and $E^{* 4}$ is the operator of $E^{* 2} E^{* 2}$, which is defined as

$E^{* 2}=\frac{\partial^{2}}{\partial r^{* 2}}+\frac{\sin \theta}{r^{* 2}} \frac{\partial}{\partial \theta}\left(\frac{1}{\sin \theta} \frac{\partial}{\partial \theta}\right)$.

Corresponding boundary conditions are

$$
\begin{aligned}
& \psi^{*}=0, \quad \frac{\partial \psi^{*}}{\partial r^{*}}=0 \quad \text { at } r^{*}=1, \\
& \psi^{*}=\frac{1}{2} r^{* 2} U^{*} \sin ^{2} \theta, \quad \text { at } r^{*}=b / a, \\
& E^{2} \psi^{*}=0 \quad \text { at } r^{*}=b / a .
\end{aligned}
$$

Equation (20) suggests no slip on the particle surface, and Eq. (21) states that the fluid is flowing toward the stationary colloid with a relative scaled velocity of $U^{*}$. Equation (22) states that the virtual surface is curlless, as proposed by the Kuwabara unit-cell model [33].

Having done the mathematical manipulations mentioned above, we end up with governing equations identical to those in electrophoresis, except for the boundary conditions in Eqs. (14) and (17) at the outer virtual surface. As pointed out by Dukhin and Deryagin, diffusiophoresis and electrophoresis have a common base. They are two cases of a more general phenomenon, namely particle movement under the influence of an external electrochemical potential gradient. When an external electric field is present, while an external gradient of concentration is absent, the movement is called electrophoresis. When there is an external concentration gradient, while an external electric field is absent, the movement is called diffusiophoresis. Moreover, both phenomena are described by the identical electrokinetic equation [13]. Note that this concentration gradient in diffusiophoresis can be huge near a surface reaction area at times, as pointed out by Prieve [16] and Smith and Prieve [17], which actually drives the colloids.

The details of the above derivations can be found elsewhere, such as Chu and co-workers [8], among our other previous publications. We now follow the same approach as that of Prieve and Roman [22], who assumed the applied concentration gradient to be very weak. Under this condition, the problem can be simplified to a linear system, allowing decomposition into two virtual subproblems $[6,22]$. In the first problem the spherical particle moves with a velocity in the absence of the applied concentration gradient, where as in the second problem the spherical particle is somehow held stationary when the concentration gradient is applied. If the corresponding forces acting on the surfaces of spherical particles for the two problems are $F_{1}$ and $F_{2}$, respectively, then $F_{1}=f_{1}^{\prime}\left(\nabla^{*} n_{0}^{*}\right)$ and $F_{2}=f_{2}^{\prime} U^{*}$, where $f_{1}^{\prime}$ and $f_{2}^{\prime}$ are proportional constants [22]. Therefore, the diffusiophoretic mobility $U_{\mathrm{m}}^{*}$ can be written as

$U_{\mathrm{m}}^{*}=\frac{U^{*}}{E_{z}^{*}}=\frac{f_{1}^{\prime}}{f_{2}^{\prime}}$.

Note that both $F_{1}$ and $F_{2}$ are computed given the values of $\nabla^{*} n_{0}^{*}$ and $U^{*}$; thus the constants $f_{1}^{\prime}$ and $f_{2}^{\prime}$ are determined straightforwardly, and $U_{\mathrm{m}}^{*}$ can be calculated via Eq. (23).

\section{Results and discussion}

A pseudo-spectral method based on the Chebyshev polynomial $[38,39]$ is adopted for the solution of the governing equations, subject to the associated boundary conditions. Details of the pseudo-spectral method employed in analyzing electrokinetic phenomena can be found elsewhere, such as Lee and co-workers $[8,40]$. It proves to be a very powerful and suitable method for the fields of interest.

Fig. 2 shows the calculated normalized diffusiophoretic mobilities as a function of surface potential $\phi_{\mathrm{r}}$ when $\beta=0, \alpha=1$, and $P e_{1}=P e_{2}=0.26(\mathrm{KCl})$. The characteristic value of diffusiophoretic mobility is chosen to be

$U^{0}=\frac{\varepsilon}{\mu a}\left(\frac{k T}{z_{1} e}\right)^{2} \nabla^{*} n_{0}^{*}$.

Note that we use the same characteristic value as Keh and Wei in 2002 [31]. Compared with the results of Wei and Keh [30] and Keh and Wei [31] for $\kappa a=1$ and 10 (dashed line in Fig. 2), which is the limiting case of ours at low zeta potential and very thin double layer, we reproduce their results exactly when $\phi_{\mathrm{r}}$ is low enough, indicating the accuracy of our results. As $\phi_{\mathrm{r}}$ increases, however, deviation between our results and theirs increases, indicating that the low zeta potential approach runs into problems for $\left|\phi_{\mathrm{r}}\right|$ higher than 1 approximately. This observation is consistent with our general conclusion in the previous study of electrophoresis $[8,40]$. 


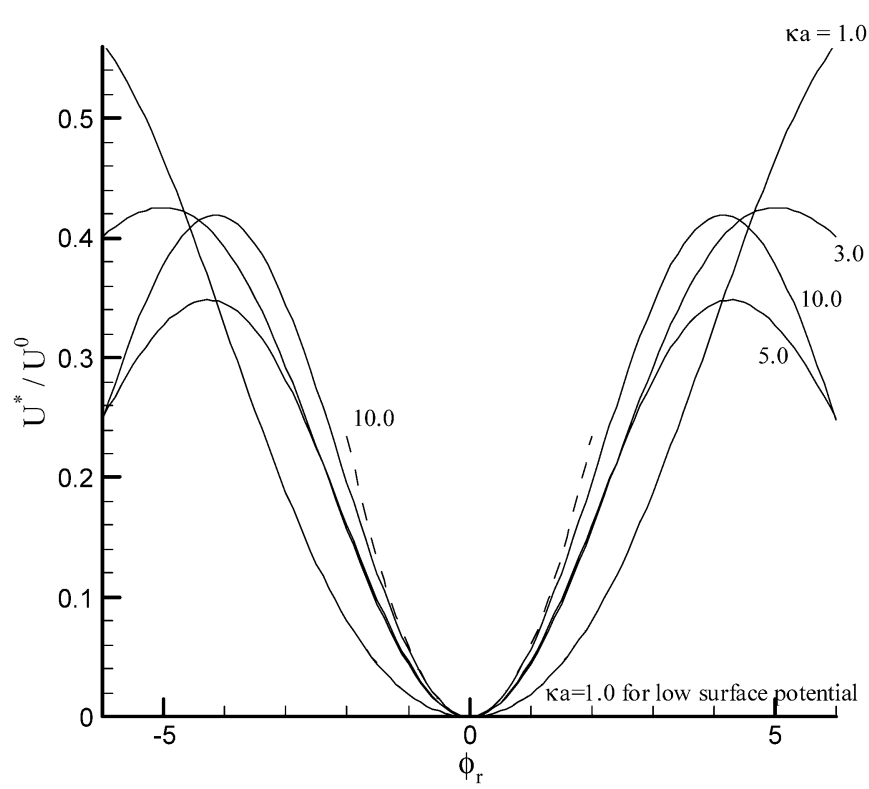

Fig. 2. Variation of scaled diffusiophoretic mobility $\left(U^{*} / U^{0}\right)$ as a function of $\phi_{\mathrm{r}}$ at various values of $\kappa a$ when $\varphi=0.1, \beta=0$, and $\alpha=1$. Dashed line, low-surface-potential case.

We also find that the mobilities for all $\kappa a$ are even functions of $\phi_{\mathrm{r}}$, which is exactly the same observation as for Prieve and Roman [22], Keh and Wei [31], or Wei and Keh [43]. According to their analysis, diffusiophoresis of the particle comes solely from chemiphoresis since the diffusion velocities are identical for cations and anions $(\beta=0)$. The term "chemiphoresis" implies that the motion of a charged particle is due to the nonuniform adsorption of counterions within the electrical double layer [31,43]. It reflects the governing equations and corresponding boundary conditions in two ways: the contribution from the disturbed electric potential, $\delta \phi^{*}$, is zero in Eq. (12), and the Peclet numbers are identical in Eq. (15) (here we set $P e_{1}=P e_{2}=0.26$, that is, a $\mathrm{KCl}$ solution). Keh's study focused on low zeta potential, whereas Prieve's focused on dilute colloidal suspensions. In the present paper, we extend it to concentrated colloidal dispersions and conclude that it is always an even function of $\phi_{\mathrm{r}}$ at arbitrary $\kappa a$, whether for dilute or concentrated dispersions. Most important of all, we find that the diffusiophoretic mobility does not increase all the way with increasing $\phi_{\mathrm{r}}$. It actually reaches a local maximum first, and then decreases. In other words, when $\phi_{\mathrm{r}}$ exceeds a certain threshold value, the mobility of the colloid no longer increases monotonously with it. This is contrary to the reports made before by Keh and Wei [31], where they studied the limiting case of low zeta potential and thin double layer. Under these assumptions, they claimed that mobility increased monotonically with $\phi_{\mathrm{r}}(\beta=0)$. We compared with their results and found that the low-zeta-potential approach, which linearized the Poisson-Boltzmann equation, is applicable only for $\left|\phi_{\mathrm{r}}\right|<1$. Once above this range, the deviation becomes more and more significant. For example, for $\left|\phi_{\mathrm{r}}\right|=3$ and $\kappa a=10$, we find that their predictions extrapolated beyond $\left|\phi_{\mathrm{r}}\right|=2$ in their paper can be as high as $40 \%$ overestimated, compared with the actual mobility based on our general approach, which allows arbitrarily

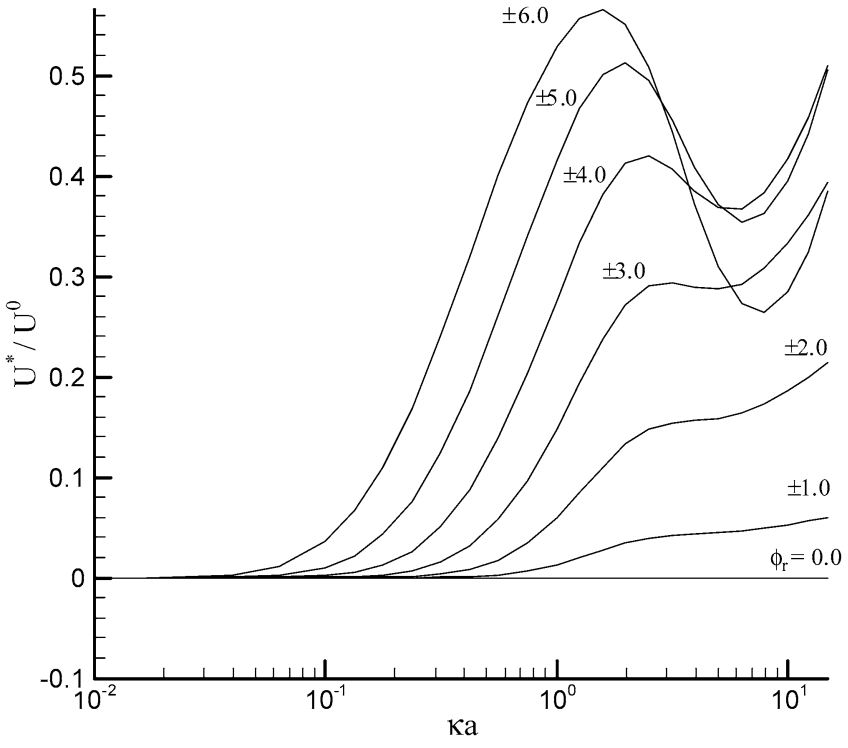

Fig. 3. Variation of scaled diffusiophoretic mobility $\left(U^{*} / U^{0}\right)$ as a function of $\kappa a$ at various values of $\phi_{\mathrm{r}}$ when $\varphi=0.125, \beta=0$, and $\alpha=1$.

high values of zeta potential. At extremely cases as $\left|\phi_{\mathrm{r}}\right| \geqslant 5$, the deviation may even be severalfold.

However, the diffusiophoretic mobility becomes somewhat irregular between $\kappa a=3$ and 5 in Fig. 2. To explain what happens there, we use $\kappa a$ as abscissa in Fig. 3 for convenience. In Fig. 3, we see similar behavior over a certain range of $\kappa a$. Briefly speaking, higher $\kappa a$ results in greater mobility of the particle because higher $\kappa a$ means higher ion concentration $n_{j 0}$, according to Eq. (8). In other words, as $\kappa a$ increases, the amount of electrolytes increases accordingly in the vicinity of the colloid surface; hence the electric force exerted upon it increases simultaneously. However, Malkin, Korotkova, and their collaborators $[44,45]$ showed that the rate of diffusiophoresis of polystyrene latex particles decreases and then changes direction with the increase of electrolyte concentration. This was the first time that the inversion of diffusiophoresis direction was predicted. They also suggested that concentration polarization of the double layer strongly influences the direction of particle movement [44]. In our study, we also find the appearance of both a local maximum and minimum over some range of $\kappa a$. This phenomenon, analyzed in depth by Dukhin [24], may arise from the polarization of the double layer surrounding the particle, which induces a microscopic electroosmosis slip opposing the normal diffusiophoretic motion. Although the gradient of potential increases due to the reduction of doublelayer thickness (larger $\kappa a$ ), which implies a stronger driving force for particle motion, the opposing electric force becomes stronger also. Comparing the variation of electric force $F_{\mathrm{E}}^{*}$ for various $\phi_{\mathrm{r}}$, as shown in Fig. 4, we find that the variations of $F_{\mathrm{E}}^{*}$ and mobility with $\kappa a$ have similar trends [8]. According to this analysis, the ultimate behavior of the diffusiophoretic mobility is mainly due to the dominant effect of $F_{\mathrm{E}}^{*}$. Lee et al. [8] also reported this result in their study of electrophoresis. However, the concentration polarization of the double layer here produces a coupled mass and charge flow near the surface of the particle. This makes the motion of the particle much more complicated 


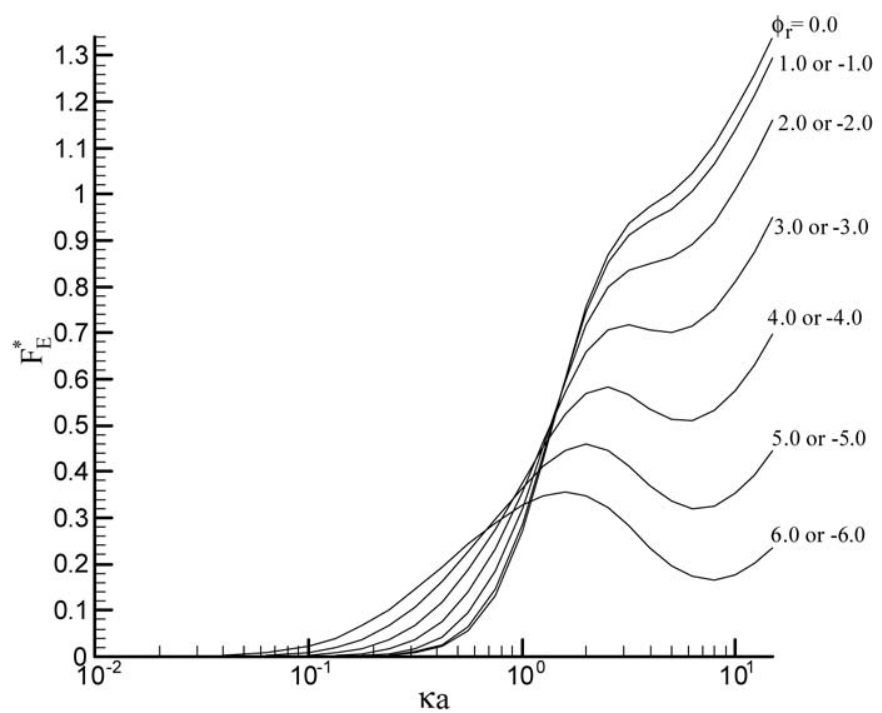

Fig. 4. Variation of scaled electric force $F_{\mathrm{E}}^{*}$ on the particle surface as a function of $\phi_{\mathrm{r}}$ at various values of $\kappa a$ when $\varphi=0.125, \beta=0$, and $\alpha=1$.

at high zeta potential or medium double-layer thickness. Therefore, even taking no consideration of the effect of discrepant diffusion speeds for cations and anions, it is still possible that the movement of the colloids may change direction more than once, as we actually observe from our calculation results. The cause of this phenomenon is the polarization effect, as we have elaborated above. Moreover, note that the mobilities for each $\kappa a$ are all symmetric functions of $\phi_{\mathrm{r}}$ in Fig. 3. If the diffusion velocities are different for cations and anions, i.e., $\beta \neq 0$, an induced electroosmotic flow will appear near the particle surface. The resulting velocity direction of the colloid is much more complicated, as it is determined by the competing chemiphoretic and electrophoretic components under the circumstances [24].

We also plot equilibrium potential $\phi_{\mathrm{e}}^{*}$ distribution at various surface potentials $\phi_{\mathrm{r}}$ in Figs. 5 and 6. We find qualitatively similar contours between Figs. 5 and 6. The major difference is the quantitative value of $\phi_{\mathrm{e}}^{*}$. On the other hand, a comparison between cases of thick ( $\kappa a=0.01)$ and thin $(\kappa a=7.3)$ doublelayer for contour plots of $\phi_{\mathrm{e}}^{*}$ is also shown in Fig. 5. In Fig. 5, it is obvious that the distribution for $\phi_{\mathrm{e}}^{*}$ is a strong function of double-layer thickness. The electric double-layer represents the range of surface potential that can affect the ion distribution. When the thickness of double layer becomes greater than the radius of virtual surface, indicated by $\kappa a$ smaller than 1 , the double-layer overlapping becomes significant. Under the same value of zeta potential, we find a steeper gradient of $\phi_{\mathrm{e}}^{*}$ near the particle surface in Fig. $5 b$, where $\kappa a$ is large, compared with Fig. 5a, where $\kappa a$ is small. Therefore, the corresponding mobility in Fig. 5 and the electrical body force in Fig. 4 of $\kappa a=7.3$ are larger than those at $\kappa a=0.01$. This result also implies that $\kappa a$ has a greater influence on the distribution of $\phi_{\mathrm{e}}^{*}$ than $\phi_{\mathrm{r}}$ does. The corresponding comparison for the scaled counterion distribution $n_{2}^{*}$ is shown in Fig. 7. When $\kappa a=0.01$, a lot of counterions are attracted into the cell, by the particle surface potential, resulting in a large area of double layer. At small $\kappa a$, we observe that the distribution of $n_{2}^{*}$ forms concen-

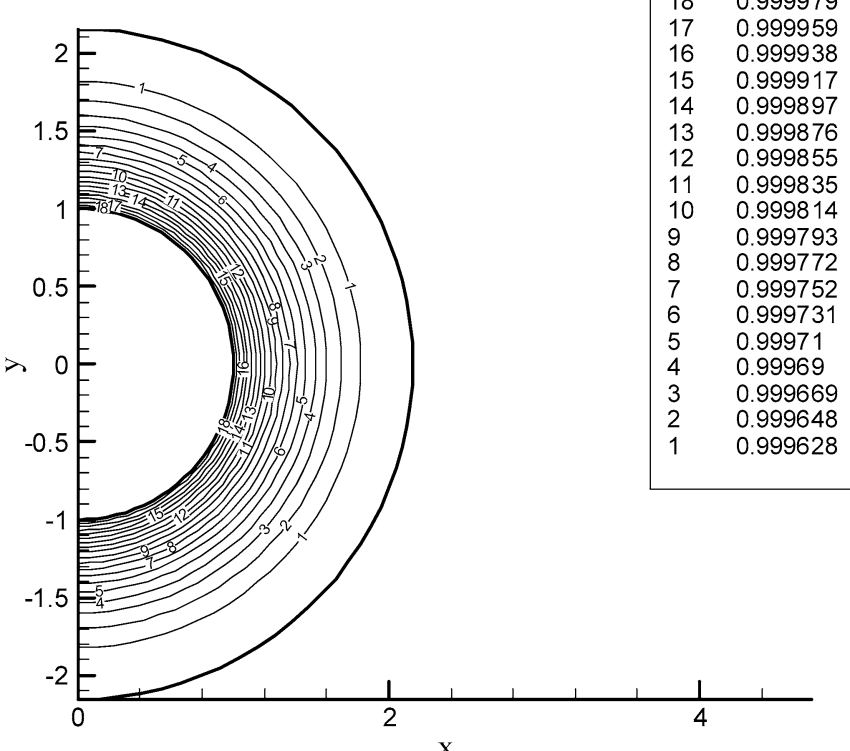

(a)

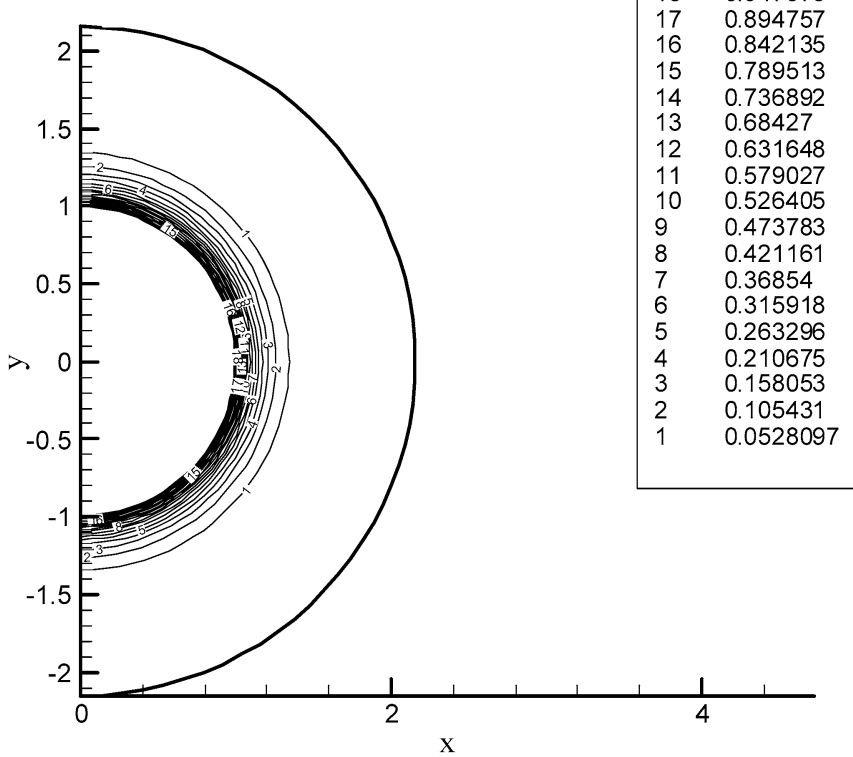

(b)

Fig. 5. Contours of scaled equilibrium potential $\phi_{\mathrm{e}}^{*}$ at two levels of $\kappa a$ when $\varphi=0.1, \beta=0, \phi_{\mathrm{r}}=3.0$, and $\alpha=1$ : (a) $\kappa a=0.01$, (b) $\kappa a=7.3$.

tric shells around the spherical particle. The larger the value of $n_{2}^{*}$, the closer the contour is to the particle surface. With the increase of $\kappa a$, the contour of $n_{2}^{*}$ distorts slightly toward the side of low ion concentration. If we compare the value of $n_{2}^{*}$ at $(0.7,-0.75)$ and $(0.7,0.75)$ in Fig. $7 \mathrm{~b}$, we find a small deviation of ionic concentration within the double layer. It is 0.984072 at $(0.7,-0.75)$ but 0.983046 at $(0.7,0.75)$. As we have explained earlier, this is because the polarization effect reaches its peak at medium $\kappa a$, since the double-layer overlapping eventually disappears. The different value of $n_{2}^{*}$ represents the possibility that the profile of double layer may be a little distorted toward the opposite direction from the particle move- 

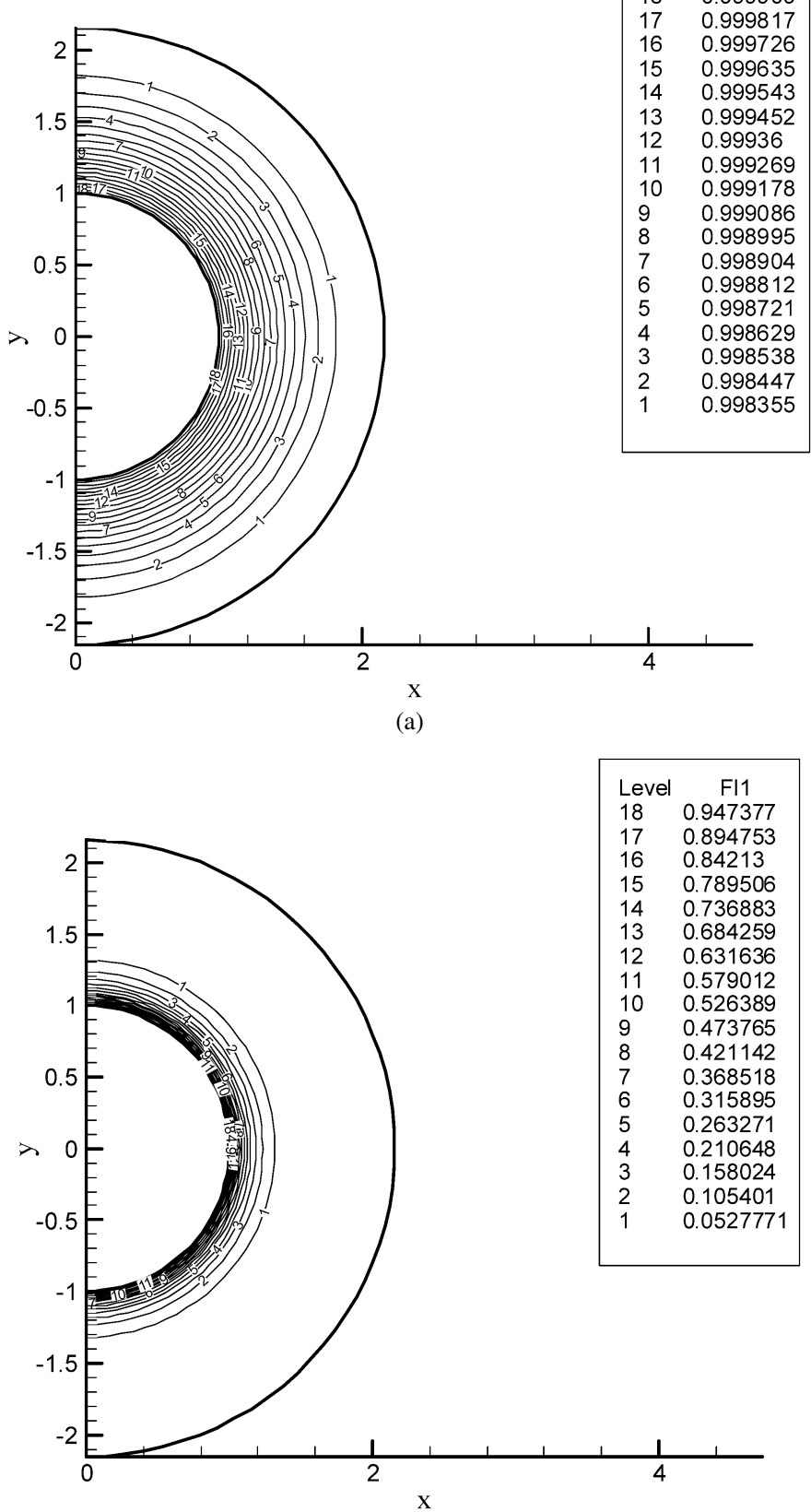

(b)

Fig. 6. Contours of scaled equilibrium potential $\phi_{\mathrm{e}}^{*}$ at two levels of $\kappa a$ when $\varphi=0.1, \beta=0, \phi_{\mathrm{r}}=5.0$, and $\alpha=1:$ (a) $\kappa a=0.01$, (b) $\kappa a=7.3$.

ment. This nonuniform distribution of ions induces an electric force retarding particle movement [8]. Furthermore, if we set $\phi_{\mathrm{r}}=3$ on the particle surface, more anions accumulate near the particle surface due to the electrostatic force. Consequently, the value of $n_{2}^{*}$ is always greater than zero within the double layer. For completeness, corresponding streamlines around the particle are also shown in Fig. 8. It is very interesting to note that when $\kappa a=0.01$, the mobility of colloidal particles and the velocity of the fluid are lower than for $\kappa a=7.3$. Therefore, the change of the value of the stream function is smaller in Fig. 8a than in Fig. 8b, indicating that the particle moves in

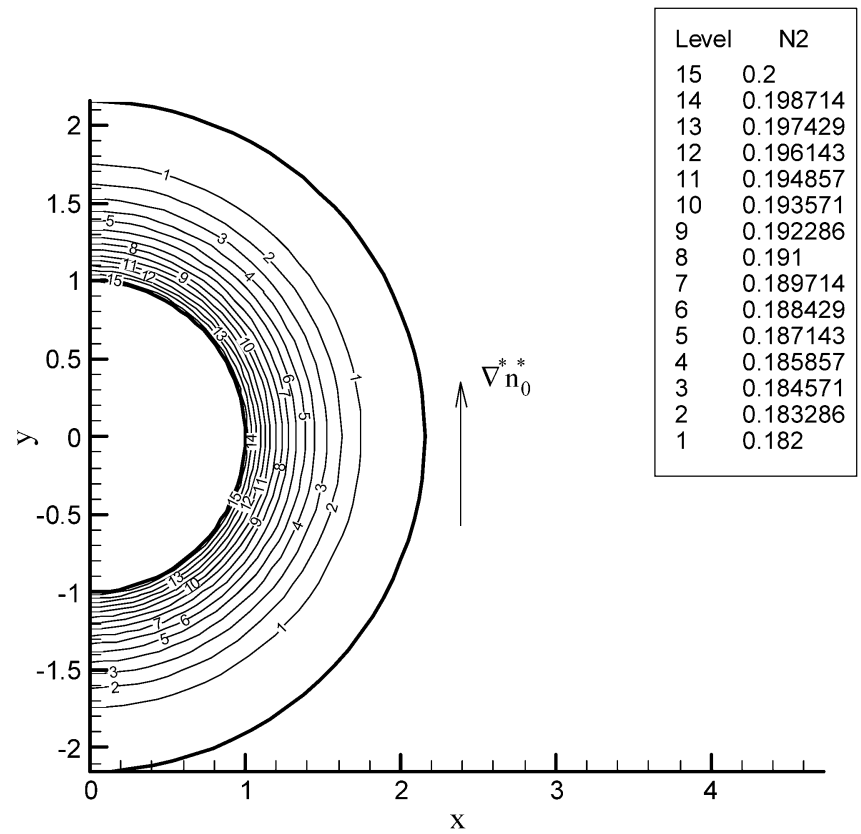

(a)

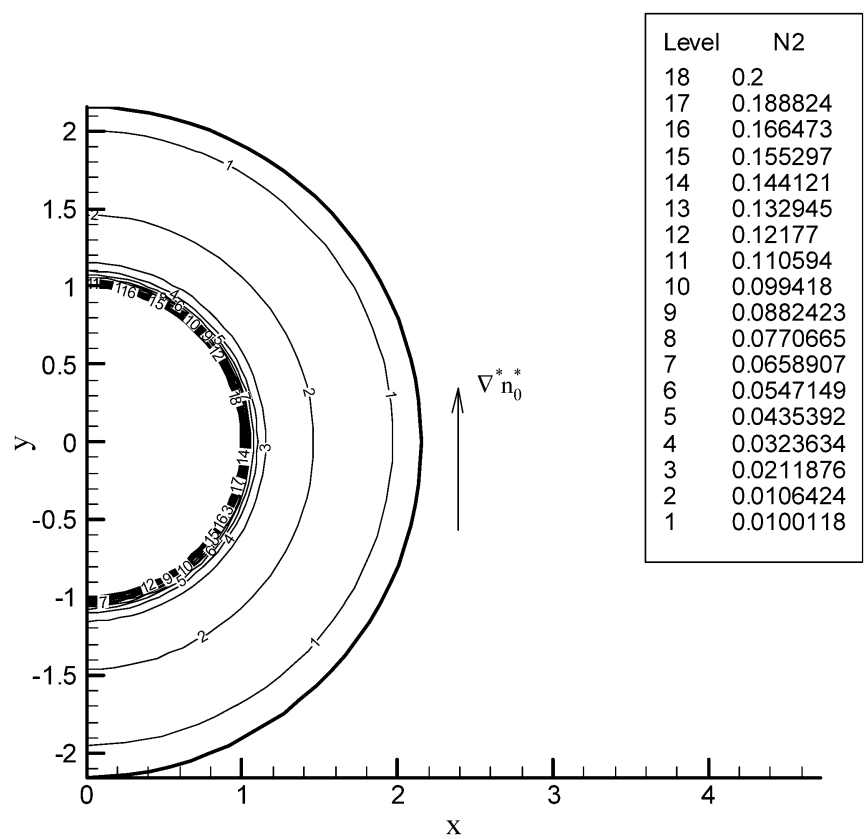

(b)

Fig. 7. Contours of $n_{2}^{*}$ at two levels of $\kappa a$ when $\varphi=0.1, \beta=0, \phi_{\mathrm{r}}=3.0$, and $\alpha=1$ : (a) $\kappa a=0.01$, (b) $\kappa a=7.3$.

the same direction of $\nabla^{*} n_{0}^{*}$ relative to the fluid on the virtual surface.

Finally, the influence of the particle concentration, measured by the volume fraction $\varphi=(a / b)^{3}$ of the particle in the cell, on the diffusiophoretic mobility in general is presented in Figs. 9 and 10. Fig. 9 shows the variation of scaled diffusiophoretic mobility as a function of volume fraction $\varphi$ at various $\phi_{\mathrm{r}}$, for a representative case of $\kappa a=1.0$. Clearly the mobility decreases accordingly when the dispersion gets more and more concentrated. This is fully expected because the higher the concentration of particles, the more significant the interaction among 

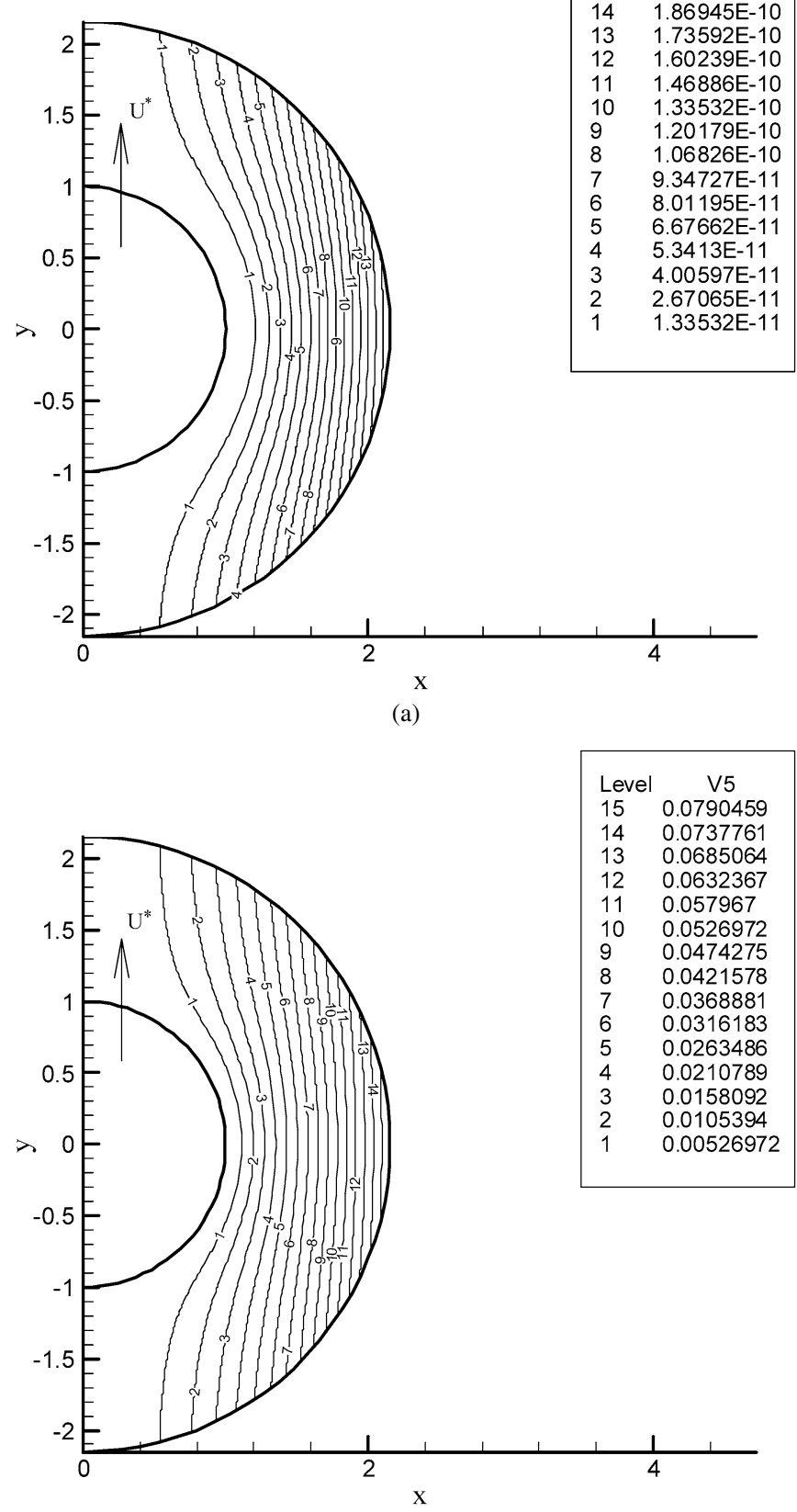

(b)

Fig. 8. Contours of stream function at two levels of $\kappa a$ when $\varphi=0.1, \beta=0$, $\phi_{\mathrm{r}}=3.0$, and $\alpha=1$ : (a) $\kappa a=0.01$, (b) $\kappa a=7.3$.

particles in the neighborhood, and thus the more important the steric hindrance for the fluid flow during diffusiophoresis. Here the steric hindrance refers to the resistance of fluid motion owing to the existence of neighboring particles. Surely it is difficult to swim fast in a crowded swimming pool. In addition, we also find that the steric hindrance delays the dominance of the polarization effect on the diffusiophoretic mobility, as shown in Figs. 9 and 10, where the occurrence of local maximum shifts to the right side along the $\phi_{\mathrm{r}}$ or $\kappa a$ axis. The shift may be so profound that the local maximum, caused by the polarization effect explained earlier, is pushed beyond the scope of the

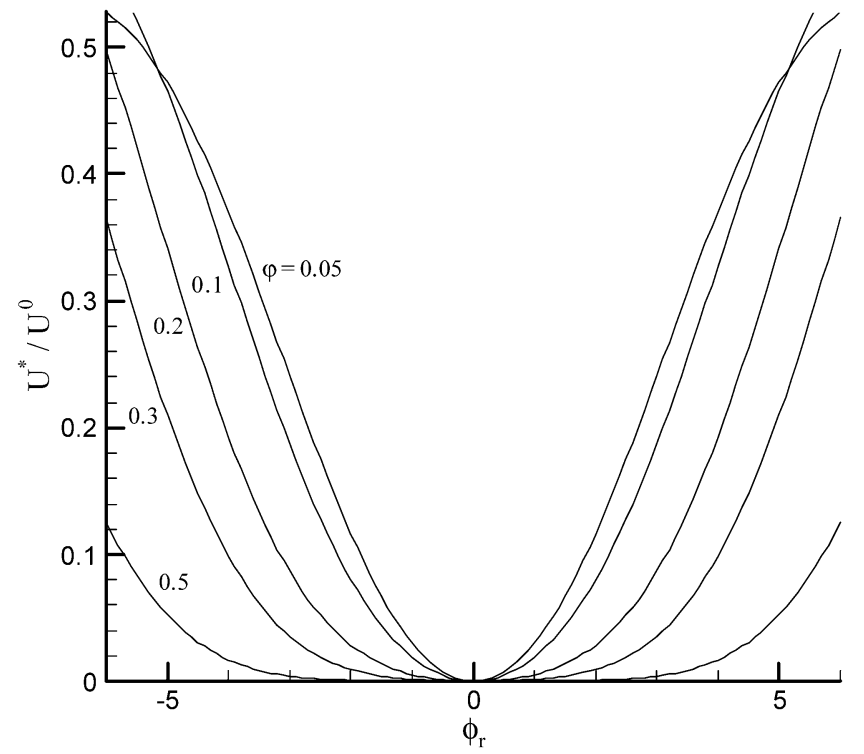

Fig. 9. Variation of scaled diffusiophoretic mobility $\left(U^{*} / U^{0}\right)$ as a function of $\phi_{\mathrm{r}}$ at various values of $\varphi$ when $k a=1.0, \beta=0$, and $\alpha=1$.

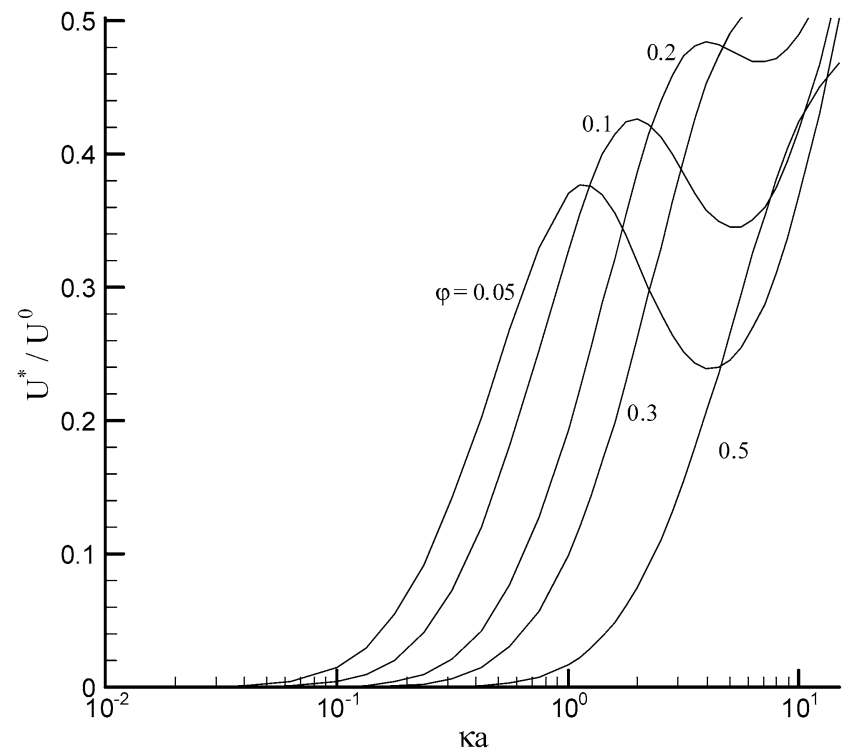

Fig. 10. Variation of scaled diffusiophoretic mobility $\left(U^{*} / U^{0}\right)$ as a function of $\kappa a$ at various values of $\varphi$ when $\phi_{\mathrm{r}}=4.0, \beta=0$, and $\alpha=1$.

figure. Though the specific case presented is for $\phi_{\mathrm{r}}=4.0$ in Fig. 10, this delay or shift-to-the-right behavior is typical in our other calculations. This is again due to the crowd of the particles when the volume fraction is increased. When the double layer around each particle is thick (small $\kappa a$ ), the overlapping is very profound at concentrated dispersions (large volume fraction). Therefore, the polarization effects are somewhat offset by one another, making the system shift toward the uniform phase.

\section{Conclusions}

We investigated here diffusiophoresis phenomena in concentrated colloidal dispersions at arbitrary zeta potentials and double-layer thicknesses. When the diffusion coefficients of 
cations and anions in the electrolyte solution are identical, each other, the diffusiophoretic mobility is found to exhibit both a local maximum and a local minimum with increasing $\phi_{\mathrm{r}}$ and $\kappa a$, due to the impact of polarization effect on the electric force exerted on the particle. This is similar to the corresponding case of dilute colloidal suspensions at high zeta potential reported in Refs. [22,24]. The previous simplified model for low zeta potential and very thin double layer is acceptable only when $\left|\phi_{\mathrm{r}}\right|<1$, and it overestimated the actual magnitude of mobility [31]. The deviation gets more and more severe as $\phi_{\mathrm{r}}$ or $\kappa a$ increases. Moreover, the particle velocity may not even move in the same direction as that of the concentration gradient. In addition, we compared the equilibrium electrical potentials and ionic concentration difference at various double layer thickness. We showed that the ion distribution around the particle, which determines the diffusiophoretic mobility, is affected by factors such as the double-layer overlapping, ion concentration gradient, and polarization effect. Finally, we find that the influence of steric hindrance on potential distribution as well as fluid flow must be taken into account as the colloidal dispersions become more and more concentrated. Overall, we conclude that the polarization effect by far is the most important factor in the determination of the colloid mobility.

\section{References}

[1] R.J. Hunter, Foundations of Colloid Science, vols. I-II, Clarendon, Oxford, 1989.

[2] J.H. Masliyah, Electrokinetic Transport Phenomena, Alberta Oil Sands Technology and Research Authority, Edmonton, 1994.

[3] M. Smoluchowski, Z. Phys. Chem. Stochiometr. Verwandtschaftslehre 92 (1918) 129.

[4] D.C. Henry, Proc. R. Soc. London Ser. A 133 (1931) 106.

[5] R.W. O’Brien, R.J. Hunter, Can. J. Chem. Rev. Can. Chim. 59 (1981) 1878.

[6] R.W. O’Brien, L.R. White, J. Chem. Soc. Faraday Trans. 274 (1978) 1607.

[7] H. Ohshima, J. Colloid Interface Sci. 189 (1997) 376.

[8] E. Lee, J.W. Chu, J.P. Hsu, J. Colloid Interface Sci. 209 (1999) 240.

[9] E. Lee, Y.S. Lee, J.P. Hsu, J. Colloid Interface Sci. 223 (2000) 223.
[10] B.V. Deryagin, S.S. Dukhin, A.A. Korotkova, Kolloidn. Zh. 23 (1961) 53.

[11] B.V. Deryagin, S.S. Dukhin, N.N. Rulev, Colloid J. USSR 40 (1978) 531.

[12] A.A. Korotkova, B.V. Deryagin, Kolloidn. Zh. 52 (1991) 861.

[13] S.S. Dukhin, B.V. Deryagin, Surface and Colloid Science, vol. 7, Wiley, New York, 1974.

[14] J.L. Anderson, Physicochem. Hydrodynam. 1 (1980) 51.

[15] D.C. Prieve, Adv. Colloid Interface Sci. 16 (1982) 321.

[16] R.E. Smith, D.C. Prieve, Chem. Eng. Sci. 37 (1982) 1213.

[17] D.C. Prieve, J.L. Anderson, J.P. Ebel, M.E. Lowell, J. Fluid Mech. 148 (1984) 247.

[18] J.P. Ebel, J.L. Anderson, D.C. Prieve, Langmuir 4 (1988) 396.

[19] W.J. Lechnick, J.A. Joseph, J. Colloid Interface Sci. 102 (1984) 71.

[20] W.J. Lechnick, J.A. Joseph, J. Colloid Interface Sci. 104 (1985) 406.

[21] G.A. Denisov, N.I. Zharkikh, Kolloidn. Zh. 53 (1991) 9.

[22] D.C. Prieve, R. Roman, J. Chem. Soc. Faraday Trans. 283 (1987) 1287.

[23] E.A. Boucher, J. Colloid Interface Sci. 129 (1989) 575.

[24] S.S. Dukhin, Adv. Colloid Interface Sci. 44 (1993) 1.

[25] J.C. Baygents, D.A. Saville, Physicochem. Hydrodynam. 10 (1988) 543.

[26] S. Misra, S. Varanasi, P.P. Varanasi, Macromolecules 23 (1990) 4258.

[27] M. Kosmulski, E. Matijevic, J. Colloid Interface Sci. 150 (1992) 291.

[28] H.J. Keh, J.S. Jan, J. Colloid Interface Sci. 183 (1996) 458.

[29] H.J. Keh, J.H. Hsu, J. Colloid Interface Sci. 221 (2000) 210.

[30] Y.K. Wei, H.J. Keh, Langmuir 17 (2001) 1437.

[31] H.J. Keh, Y.K. Wei, J. Colloid Interface Sci. 248 (2002) 76.

[32] J. Happel, AIChE J. 4 (1958) 197.

[33] S. Kuwabara, J. Phys. Soc. Jpn. 14 (1959) 527.

[34] H.J. Keh, H.C. Ma, Colloids Surf. A Physicochem. Eng. Aspects 233 (2004) 87.

[35] H.J. Keh, H.C. Ma, Langmuir 21 (2005) 5461.

[36] P. Staffeld, J. Quinn, J. Colloid Interface Sci. 130 (1989) 69.

[37] P. Staffeld, J. Quinn, J. Colloid Interface Sci. 130 (1989) 89.

[38] B.A. Finlayson, Nonlinear Analysis in Chemical Engineering, McGrawHill, London/New York, 1980.

[39] C. Canuto, M.Y. Hussaini, A. Quarteroni, T.A. Zang, Spectral Methods in Fluid Dynamics, Springer-Verlag, Berlin, 1988.

[40] J.W. Chu, W.H. Lin, E. Lee, J.P. Hsu, Langmuir 17 (2001) 6289.

[41] S.S. Dukhin, Adv. Colloid Interface Sci. 61 (1995) 17.

[42] A.V. Delgado, F. González-Caballero, R.J. Hunter, L.K. Koopal, J. Lyklema, IUPAC Techn. Report 77 (2005) 1753.

[43] H.J. Keh, Y.K. Wei, Langmuir 16 (2000) 5289.

[44] E.S. Malkin, A.S. Dukhin, Kolloidn. Zh. 44 (1982) 254.

[45] A.A. Korotkova, N.V. Churaev, B.V. Deryagin, Kolloidn. Zh. 52 (1991) 197. 